\title{
Effect of budesonide on fibroblast-mediated collagen gel contraction and degradation
}

This article was published in the following Dove Press journal:

Journal of Inflammation Research

26 Febraury 2013

Number of times this article has been viewed

\author{
Qiuhong Fang ${ }^{1,2}$ \\ Nancy A Schulte 3 \\ Huijung Kim ${ }^{4}$ \\ Tetsu Kobayashi ${ }^{5}$ \\ Xingqi Wang ${ }^{2}$ \\ Anna Miller-Larsson ${ }^{6}$ \\ Elisabet Wieslander ${ }^{6}$ \\ Myron L Toews ${ }^{3}$ \\ Xiangde Liu $^{2}$ \\ Stephen I Rennard ${ }^{2}$ \\ 'Pulmonary and Critical Care \\ Department, Beijing Shijitan \\ Hospital, Capital Medical University, \\ Beijing, People's Republic of China; \\ ${ }^{2}$ Pulmonary, Critical Care, Sleep \\ and Allergy Division, Department \\ of Internal Medicine, University of \\ Nebraska Medical Center, Omaha, NE, \\ USA; ${ }^{3}$ Department of Pharmacology \\ and Experimental Neuroscience, \\ University of Nebraska Medical \\ Center, Omaha, NE, USA; ${ }^{4}$ Pulmonary \\ and Critical Care Division, \\ WonKwang University, Kunpo \\ Medical Center, Seoul, Republic of \\ Korea; ${ }^{5}$ Mie University Graduate \\ School of Medicine, Tsu City, Japan; \\ ${ }^{6}$ AstraZeneca R\&D Molndal, Molndal, \\ Sweden
}

Background: The balance between production and degradation of extracellular matrix is crucial in maintaining normal tissue structure. This study was designed to investigate the effect of budesonide on fibroblast-mediated tissue repair and remodeling.

Methods: Using human fetal lung fibroblasts in a three-dimensional collagen gel culture system, we investigated the effect of budesonide (1-1000 $\mathrm{nM}$ ) on collagen gel contraction and degradation in the presence or absence of inflammatory cytokines (interleukin-1 $\beta$ and tumor necrosis factor $\alpha ; 5 \mathrm{ng} / \mathrm{mL}$ each) and, in order to activate latent proteases, serine protease trypsin $0.25 \mu \mathrm{g} / \mathrm{mL}$. The effects of budesonide on metalloproteinase production and activation were also investigated.

Results: Inflammatory cytokines significantly inhibited collagen gel contraction mediated by lung fibroblasts. Budesonide counteracted the effect of cytokines in a concentration-dependent manner (to $50 \%, P<0.01$ ). Budesonide $100 \mathrm{nM}$ almost completely inhibited the release and mRNA expression of metalloproteinase-1, metalloproteinase-3, and metalloproteinase-9 induced by the cytokines $(P<0.05)$. Exposure to the cytokines plus trypsin increased collagen degradation and conversion of the metalloproteinases to lower molecular weight forms corresponding to their active forms. Budesonide blocked both enhanced collagen degradation $(P<0.01)$ and suppressed trypsin-mediated conversion of cytokine-induced metalloproteinase- 9 and metalloproteinase-3 to lower molecular weight forms. Similar effects were observed with dexamethasone $1 \mu \mathrm{M}$, suggesting a class effect.

Conclusion: These findings demonstrate that budesonide directly modulates contraction of collagen gels and can decrease collagen degradation under inflammatory conditions. The mechanism of this effect is through suppressing gene expression, release, and activation of metalloproteinases. By modulating the release and activity of metalloproteinases, inhaled budesonide may be able to modify airway tissue repair and remodeling.

Keywords: metalloproteinase, budesonide, tissue remodeling

\section{Introduction}

Chronic airway inflammation contributes to the alteration of tissue structure. In the lung, this can lead to the fixed airflow limitation that is the defining feature of chronic obstructive pulmonary disease (COPD) and may play a role in asthma as well. ${ }^{1}$ In particular, the balance between extracellular matrix production and its destruction may be important in determining outcomes of tissue repair. In this regard, properly balanced and sufficient tissue repair is required to restore architectural integrity following injury. Insufficient repair may lead to emphysema, and in contrast, excessive repair may lead to fibrosis.

In the milieu of inflammation, fibroblasts can not only produce new extracellular matrix, but can also cause its degradation through release of matrix
Correspondence: Stephen I Rennard University of Nebraska Medical Center, 985910 Nebraska Medical Center, Omaha, Nebraska 68198-5910, USA $\mathrm{Tel}+\mathrm{I} 4025597313$

Fax +l 4025594878

Email srennard@unmc.edu 
metalloproteinases (MMPs). MMPs are a family of proteolytic enzymes that share structural features. ${ }^{2}$ Various MMPs have a variety of target substrates that create the potential for MMPs to regulate cellular and tissue function through a variety of mechanisms. Several MMPs have the potential to degrade extracellular matrix macromolecules, and by this mechanism have been suggested to contribute to the altered extracellular matrix that characterizes COPD. Among the 24 members of the MMP family that are present in man, in vitro studies demonstrate that MMP-1 is capable of degrading type I collagen while MMP-2 and MMP-9 are gelatinases that can degrade gelatin as well as a number of other substrates. Production of these enzymes is increased in many chronic diseases. ${ }^{3-7}$ However, their activity is regulated at several levels, including protease cleavage of latent MMPs into active lower molecular weight forms and inhibition by their biological inhibitors, tissue inhibitors of metalloproteinase (TIMPs). ${ }^{8,9}$

Budesonide is a corticosteroid that is widely used in chronic inflammatory diseases, including asthma, rhinitis, COPD, and Crohn's disease. While its anti-inflammatory effect has been extensively studied, the effect of budesonide on tissue repair and remodeling, specifically its effect on degradation of collagen, has not been fully investigated. Therefore, the current study was designed to investigate the effect of budesonide on collagen degradation as well as on MMP-1, MMP-3, and MMP-9 release, activation, and mRNA expression in human lung fibroblasts. To accomplish this, human lung fibroblasts were cultured in three-dimensional collagen gels. This culture system differs from the more commonly used method of culture on a plastic substrate. In this system, fibroblasts attach and spread along collagen fibers and separate from each other. ${ }^{10}$ The fibroblasts are capable of generating mechanical tension and can "remodel" gels by inducing their contraction. This has been used as a model for fibroblast-mediated tissue remodeling. ${ }^{10,11}$ Using this culture system, fibroblasts were stimulated with cytokines to induce MMP production and further incubated with trypsin to activate the MMPs.

\section{Materials and methods Materials}

Recombinant human interleukin (IL)- $1 \beta$ and tumor necrosis factor (TNF) $\alpha$ were purchased from R\&D Systems (Minneapolis, MN). The budesonide was a kind gift from AstraZeneca (Molndal, Sweden). Dexamethasone was purchased from Sigma (St Louis, MO). The glucocorticoids were dissolved in ethanol to a stock solution of $10^{-3} \mathrm{M}$.
The final concentration of ethanol in the cell culture media was $\leq 0.1 \%$, as described elsewhere. ${ }^{12}$ Type I collagen was extracted from rat tail tendons as previously described. ${ }^{13,14}$ Briefly, tendons were excised from rat tails, and the tendon sheath and other connective tissues were removed carefully. Repeated washing with Tris-buffered saline $(0.9 \% \mathrm{NaCl}$, $10 \mathrm{mM}$ Tris, $\mathrm{pH}$ 7.5) was followed by dehydration and sterilization with $50 \%, 75 \%, 95 \%$, and pure ethanol. Type I collagen was extracted in $6 \mathrm{mM}$ hydrochloric acid at $4^{\circ} \mathrm{C}$ for 24 hours. The supernatant was harvested by centrifugation at $2000 \mathrm{~g}$ for 2 hours at $4^{\circ} \mathrm{C}$. Protein concentration was determined by weighing a lyophilized aliquot from each lot of collagen solution. Sodium dodecyl sulfate polyacrylamide gel electrophoresis routinely demonstrated no detectable proteins other than type I collagen. The collagen extracted from rat tail tendons was stored at $4^{\circ} \mathrm{C}$ until use.

\section{Cell culture}

Human fetal lung fibroblasts (HFL-1), obtained from the American Type Culture Collection, were cultured with Dulbecco's Modified Eagle's Medium supplemented with $10 \%$ fetal calf serum, $50 \mathrm{U} / \mathrm{mL}$ penicillin, $50 \mu \mathrm{g} / \mathrm{mL}$ streptomycin, and $1 \mu \mathrm{g} / \mathrm{mL}$ fungizone. The cells were cultured in $100 \mathrm{~mm}$ tissue culture dishes. Fibroblasts were trypsinized ( $0.05 \%$ trypsin, $0.53 \mathrm{mM}$ ethylenediamine tetra-acetic acid) and used for the three-dimensional gel contraction assay.

\section{Collagen gel contraction assay}

The ability of fibroblasts to contract collagen gels was determined as described. ${ }^{15}$ Collagen gels were prepared by mixing rat tail tendon collagen, distilled water, $4 \times$ concentrated Dulbecco's Modified Eagle's Medium, and cell suspension so that the final mixture resulted in $0.75 \mathrm{mg} / \mathrm{mL}$ collagen, $4.5 \times 10^{5}$ cells/mL, and $1 \times$ Dulbecco's Modified Eagle's Medium. ${ }^{13}$ One-half milliliter of the collagen/cell mixture was plated into each well of a 24-well tissue culture plate. Gels were allowed to solidify at room temperature, usually within 15 minutes. When the gels were set, they were released into a $60 \mathrm{~mm}$ tissue culture dish containing $5 \mathrm{~mL}$ of serum-free Dulbecco's Modified Eagle's Medium with or without varying reagents, and drugs which were added simultaneously. The gels were then maintained in floating culture and size was quantified with an image analyzer daily.

\section{Gelatin zymography}

As described, conditioned media $(0.5 \mathrm{~mL})$ collected from the gel contraction assay were concentrated by ethanol precipitation and resuspended in distilled $\mathrm{H}_{2} \mathrm{O}(50 \mu \mathrm{L}){ }^{16}$ 
The samples were then added to electrophoresis sample buffer (0.5 M Tris pH 6.8, 2\% sodium dodecyl sulfate, 20\% glycerol, $0.1 \%$ bromophenol blue) and heated for 5 minutes at $95^{\circ} \mathrm{C}$. Twenty microliters of each sample was loaded into each lane of a $10 \%$ sodium dodecyl sulfate polyacrylamide gel and electrophoresed. After electrophoresis, the gels were gently soaked in $2.5 \%$ Triton $\mathrm{X}-100$ at $37^{\circ} \mathrm{C}$ twice for 30 minutes each. The gels were then incubated in metalloproteinase activation buffer $(0.06 \mathrm{M}$ Tris $\mathrm{pH} 7.5,6 \mathrm{mM}$ $\mathrm{CaCl}_{2}$, and $1 \mathrm{mM} \mathrm{ZnCl}_{2}$ ) for 18 hours at $37^{\circ} \mathrm{C}$. The gels were stained with $0.2 \%$ Coomassie blue and destained with $30 \%$ methanol and 10\% acetic acid. The gels were dried directly between cellophane sheets.

\section{Immunoblotting}

Conditioned media $(2 \mathrm{~mL})$ collected from the gel contraction assay was concentrated by ethanol precipitation and resuspended in distilled $\mathrm{H}_{2} \mathrm{O}(50 \mu \mathrm{L})$. The samples were then added to electrophoresis sample buffer $(0.5 \mathrm{M}$ Tris $\mathrm{pH} 6.8,2 \%$ sodium dodecyl sulfate, $20 \%$ glycerol, $0.1 \%$ bromophenol blue) and heated for 5 minutes at $95^{\circ} \mathrm{C}$. In order to provide a comparison of the secreted proteins, an equal volume $(30 \mu \mathrm{L})$ of each sample was loaded into each lane of a $10 \%$ sodium dodecyl sulfate polyacrylamide gel and electrophoresed. The proteins were transferred to a polyvinylidene fluoride membrane with a semidry transfer apparatus. The membrane was blocked in 5\% milk in phosphate-buffered saline-Tween at room temperature for one hour, then exposed to primary antibodies (mouse anti-human MMP-1 or MMP-3), which were subsequently detected using horseradish peroxidase-conjugated rabbit anti-mouse IgG in conjunction with an enhanced chemiluminescence detection system, as described elsewhere. ${ }^{17}$

\section{Hydroxyproline assay}

The collagen content of the gels was determined as described earlier. ${ }^{18}$ Briefly, the gels were removed from the treatment media on the appropriate day and transferred to an Eppendorf tube. The gels were centrifuged to remove remaining media and then allowed to dry overnight at room temperature. Gels were dissolved in $\mathrm{H}_{2} \mathrm{O}$ at $72^{\circ} \mathrm{C}$ for one hour, centrifuged at $1000 \mathrm{~g}$ for 5 minutes, and the supernatant was harvested for the hydroxyproline assay. Aliquots of $20 \mu \mathrm{L}$ were mixed with $30 \mu \mathrm{L}$ of $3.3 \mathrm{~N} \mathrm{NaOH}$ so that the final concentration of $\mathrm{NaOH}$ was $2 \mathrm{~N}$, and samples were hydrolyzed by autoclaving at $120^{\circ} \mathrm{C}$ for 20 minutes. Next, $450 \mu \mathrm{L}$ of 0.056 $\mathrm{M}$ chloramine- $\mathrm{T}$ was added, and oxidation was allowed to proceed for 25 minutes at room temperature. Then $500 \mu \mathrm{L}$ of Ehrlich's aldehyde reagent was added to each sample and the chromophore was developed by incubating the samples at $65^{\circ} \mathrm{C}$ for 20 minutes. Absorbance of each sample was measured at $550 \mathrm{~nm}$ by a colorometric reader.

\section{Quantification of mRNA by real time RT-PCR}

The cells were cast into collagen gels at a density of $4.5 \times 10^{5} / \mathrm{mL}$, as $2 \mathrm{~mL}$ per well of a 6 -well plate. Cells were treated with the reagents indicated for 72 hours. Total RNA was extracted with Trizol and quantified using a spectrophotometer. A two step real-time reverse transcriptase polymerase chain reaction (RT-PCR) was conducted using the following probes and primers, as we have previously described. ${ }^{19}$ Real-time PCRs of cDNA specimens were conducted in a total volume of $50 \mu \mathrm{L}$ with $1 \times$ TaqMan Master Mix (Applied Biosystems, Foster City, CA), and primers at $300 \mathrm{nM}$ and probes at $200 \mathrm{nM}$. The sequences used were as follows:

MMP-1 (forward), 5'-CGG TTT TTC AAA GGG AAT AAG TAC T-3';

MMP-1 (reverse), 5'-TCA GAA AGA GCA GCA TCG ATA TG-3';

MMP-1 (probe), 6FAM-AAT GTG CTA CAC GGA TAC CCC AAG GAC A-TAMRA;

MMP-9 (forward), 5'-AGA GAG GAG GAG GTG GTG TAA GC-3';

MMP-9 (reverse), 5'-TGA CAG GCA AGT GCT GAC TCA-3';

MMP-9 (probe), 6FAM-TT TCT CAT GCT GGT GCT GCC ACA CA-TAMRA;

MMP-3 (forward), 5'-GAT ATA AAT GGC ATT CAG TCC CTC TAT-3';

MMP-3 (reverse), 5'-CAA AGG ACAAAG CAG GAT CAC A-3';

MMP-3 (probe), 6FAM-CCT CCC CCT GAC TCC CCT GAG ACC-TAMRA.

Data were analyzed, normalized to rRNA, and expressed as fold of control following the method we have published previously. ${ }^{20}$

\section{Statistical analysis}

Individual contraction experiments included triplicate gels within an experiment for all experimental conditions. Results were always confirmed by repeating each experiment on separate occasions at least three times. Statistical comparisons were made from three separate experiments, including both within-group and between-group variance. For immunoblotting and zymography of MMPs, each sample was harvested 
from one dish containing triplicate collagen gels. Results were confirmed by repeating each experiment on separate samples at least three times. Expression of mRNAs was expressed as fold change compared with control (untreated cells) for all experiments. Group data were analyzed by oneway or two-way analysis of variance followed by Tukey's test using PRISM4 software. $P<0.05$ was considered to be statistically significant.

\section{Results}

In order to evaluate the ability of budesonide to modify fibroblast degradation and remodeling of the extracellular matrix, the fibroblasts were cast into three-dimensional collagen gels and maintained in floating culture. As previously shown, ${ }^{21}$ over three days of culture and in control conditions, the collagen gels contracted to approximately $40 \%$ of their original size (Figure 1). In the presence of the cytokines IL-1 $\beta$ and TNF $\alpha$, fibroblast-mediated collagen gel contraction was inhibited to $80 \%$ of initial size (Figure 1). Budesonide added under control conditions did not affect (Figure 1) or resulted in slight augmentation of collagen gel contraction (see Figure 5A). However, in the presence of cytokines, budesonide counteracted their effect and significantly augmented the contraction compared with cytokines alone in a concentration-dependent manner (Figure 1).

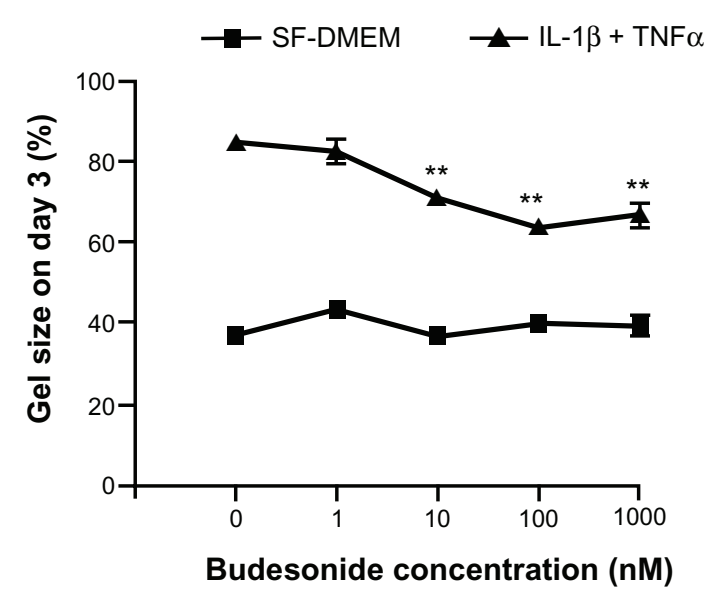

Figure I Effect of budesonide on collagen gel contraction in the presence or absence of cytokines.

Notes: Fibroblasts (HFL-I) were cast into three-dimensional collagen gels, released into SF-DMEM with or without IL-I $\beta$ and TNF $\alpha$, and then incubated for 5 days with or without budesonide. Gel size was measured daily and the data presented are for gel size on day 3. The vertical axis shows gel size expressed as percent of original size (\%); the horizontal axis shows budesonide concentration (nM). The square represents SF-DMEM; the triangle represents IL-I $\beta$ and TNF $\alpha(5 \mathrm{ng} / \mathrm{mL}$ for each cytokine). Each point is the mean \pm standard deviation of triplicate gels from a representative experiment that was repeated three times. ${ }^{*} * P<0.01$ for treatment with medium + budesonide compared with treatment comprising cytokines and budesonide by two-way analysis of variance followed by Tukey's test.

Abbreviations: SF-DMEM, serum-free Dulbecco's Modified Eagle's Medium; IL-I $\beta$, interleukin-I $\beta$; TNF $\alpha$, tumor necrosis factor $\alpha$.
After 5 days of culture, the media in which the collagen gels had been cultured were harvested and used to assess MMP release and conversion to active forms. Under control conditions (serum-free culture medium), MMP-1, MMP-3, and MMP-9 were undetectable (Figure 2A-C, respectively) whereas detectable MMP-2 was released into the culture medium corresponding to both latent $(72 \mathrm{kDa})$ and active (66 kDa) forms, which were slightly inhibited by budesonide (Figure $2 \mathrm{C}$ ). When the fibroblasts were stimulated with the cytokines IL- $1 \beta$ and TNF $\alpha$, there was significant upregulation of MMP-1, MMP-3, and MMP-9, but not MMP-2, and these were readily detected, with apparent molecular weights that corresponded to latent forms of MMP-1 (54 kDa), MMP-3 (59 kDa), and MMP-9 (92 kDa, Figure 2). Budesonide inhibited the release of MMP-1, MMP-3, and MMP-9 induced by IL-1 $\beta$ and TNF $\alpha$ (Figure 2). TIMP-1 released into the collagen gel culture medium was also assessed by enzyme-linked immunosorbent assay. Cytokines slightly but not significantly inhibited TIMP-1 release, and budesonide had no effect on TIMP-1 release (data not shown).

Next, the effect of budesonide on expression of MMP mRNAs was determined by real-time RT-PCR after 3 days of culture. As expected, IL-1 $\beta$ and TNF $\alpha$ significantly stimulated mRNA expression for MMP-1, MMP-3, and MMP-9 (Figure 3). Budesonide $100 \mathrm{nM}$ alone did not significantly affect MMP mRNA expression, but budesonide significantly ( $P<0.05$, all comparisons) inhibited expression of mRNA stimulated by IL-1 $\beta$ and TNF $\alpha$ for MMP-1, MMP-3, and MMP-9 (Figure 3A-C, respectively, $P<0.05)$.

Because MMP-1, MMP-3, and MMP-9 were released by fibroblasts in the presence of cytokines in their latent forms, trypsin was added to activate the MMPs in order to study their functional significance. As expected, the latent forms of MMP-1, MMP-3, and MMP-9 were largely converted into their corresponding active forms by trypsin (Figure 4A-C, respectively). Budesonide not only reduced production of latent MMPs in the presence of the cytokines, but also partially blocked activation of cytokine-induced MMP-3 and MMP-9 caused by trypsin (Figure 4B and C). Note that multiple bands were observed for each of the MMPs, which may correspond to differential glycosylation or to stepwise proteolytic conversion of MMPs to active forms, but are an expected result. ${ }^{2,22,23}$ Consistent with the expected consequences of MMP activation, striking effects were observed in the fibroblast-containing collagen gels incubated with the cytokines, trypsin, and/or budesonide. 


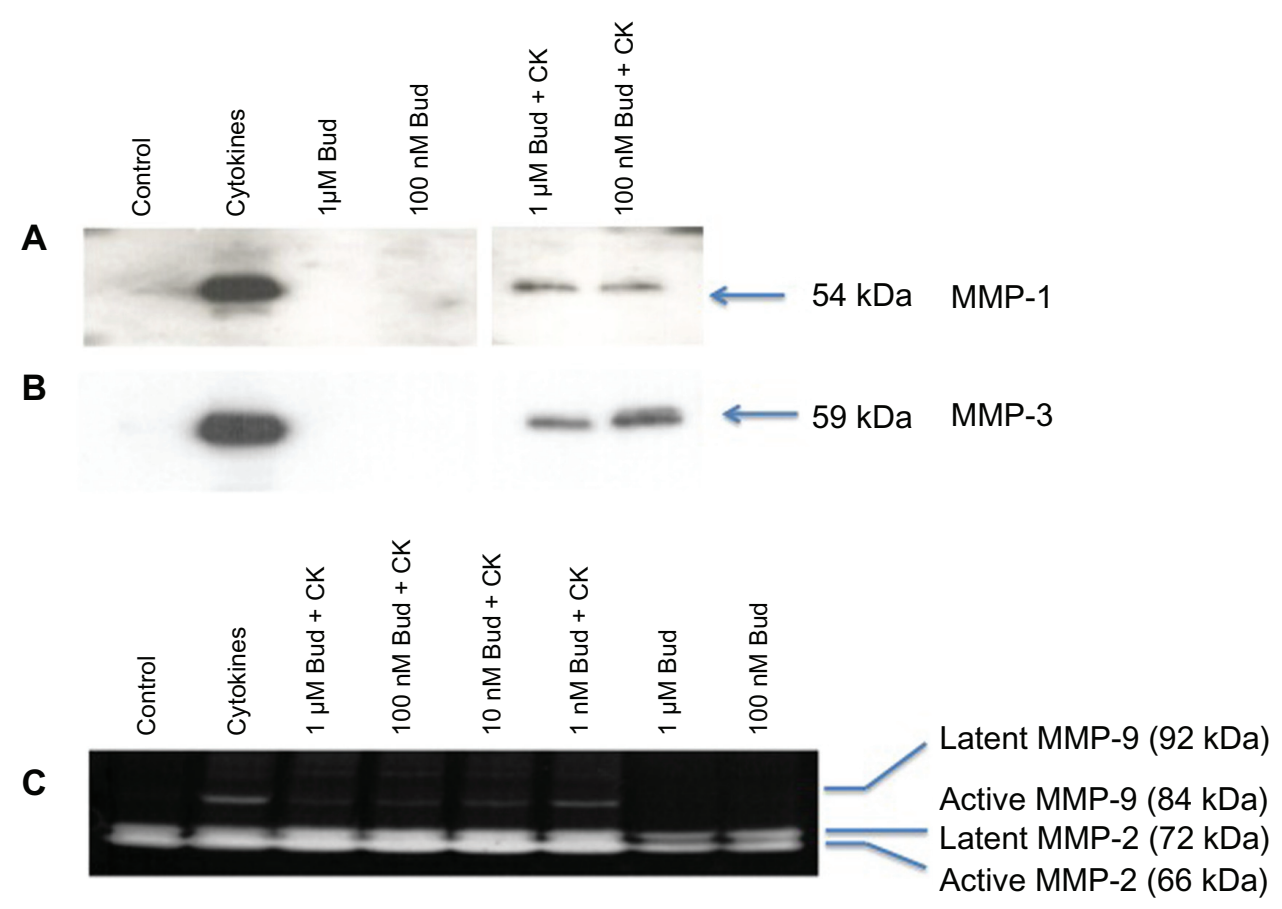

Figure 2 Budesonide inhibits release of MMP-I, MMP-2, MMP-3, and MMP-9. Culture media of Figure I were harvested on day 5 and used for zymography or immunoblot as described in the methods. (A) Immunoblotting for MMP-I. (B) Immunoblotting for MMP-3. (C) Gelatin zymography for MMP-2 and MMP-9.

Notes: The clear bands indicate gelatinase activity. Data presented are representative of three separate experiments.

Abbreviations: $\mathrm{CK}$, cytokines (interleukin- $1 \beta+$ tumor necrosis factor- $\alpha ; 5 \mathrm{ng} / \mathrm{mL}$ each); Bud, budesonide; MMP, metalloproteinase.

\section{A MMP-1}

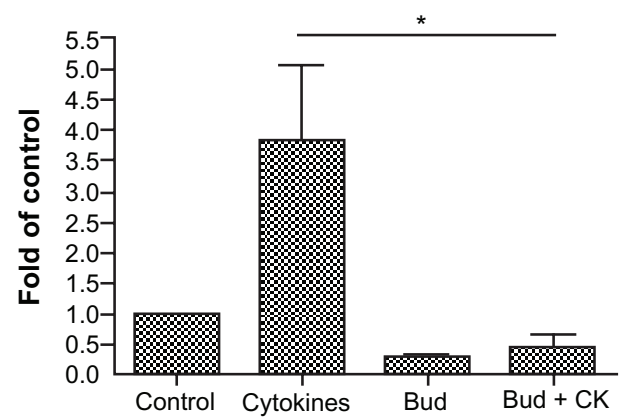

\section{B MMP-3}

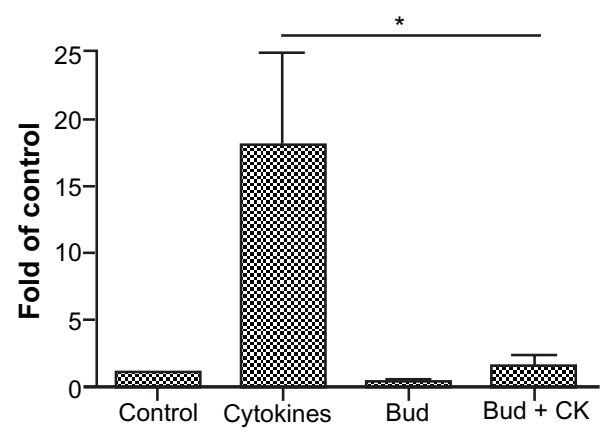

\section{MMP-9}

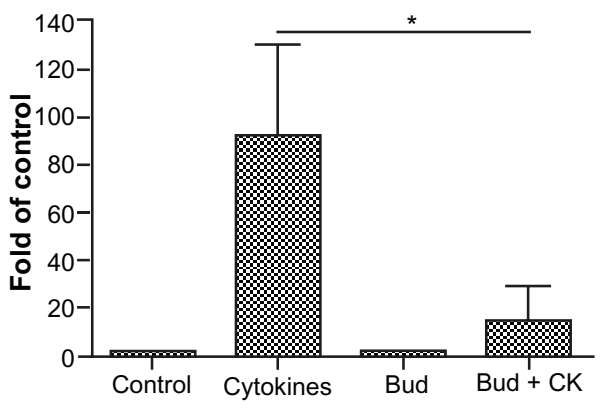

Figure 3 Inhibition of MMP mRNA expression by budesonide. Cells were cast into three-dimensional collagen gels and maintained in serum-free Dulbecco's Modified Eagle's Medium with or without cytokines and/or budesonide for 3 days. Total RNA was extracted, and real-time reverse transcriptase polymerase chain reaction was performed as described in the Methods section for MMP-I (A), MMP-3 (B), or MMP-9 (C).

Notes: The vertical axis shows mRNA level expressed as fold of control (serum free Dulbecco's Modified Eagle's Medium condition); the horizontal axis shows treatment. Data are shown as the mean \pm standard deviation for three separate experiments. *P $<0.05$ by one-way analysis of variance followed by Tukey's test for comparison of cytokines with budesonide + cytokines.

Abbreviations: CK, cytokines (interleukin-I $\beta$ + tumor necrosis factor- $\alpha ; 5 \mathrm{ng} / \mathrm{mL}$ each); Bud, budesonide $100 \mathrm{nM}$; MMP, metalloproteinase. 


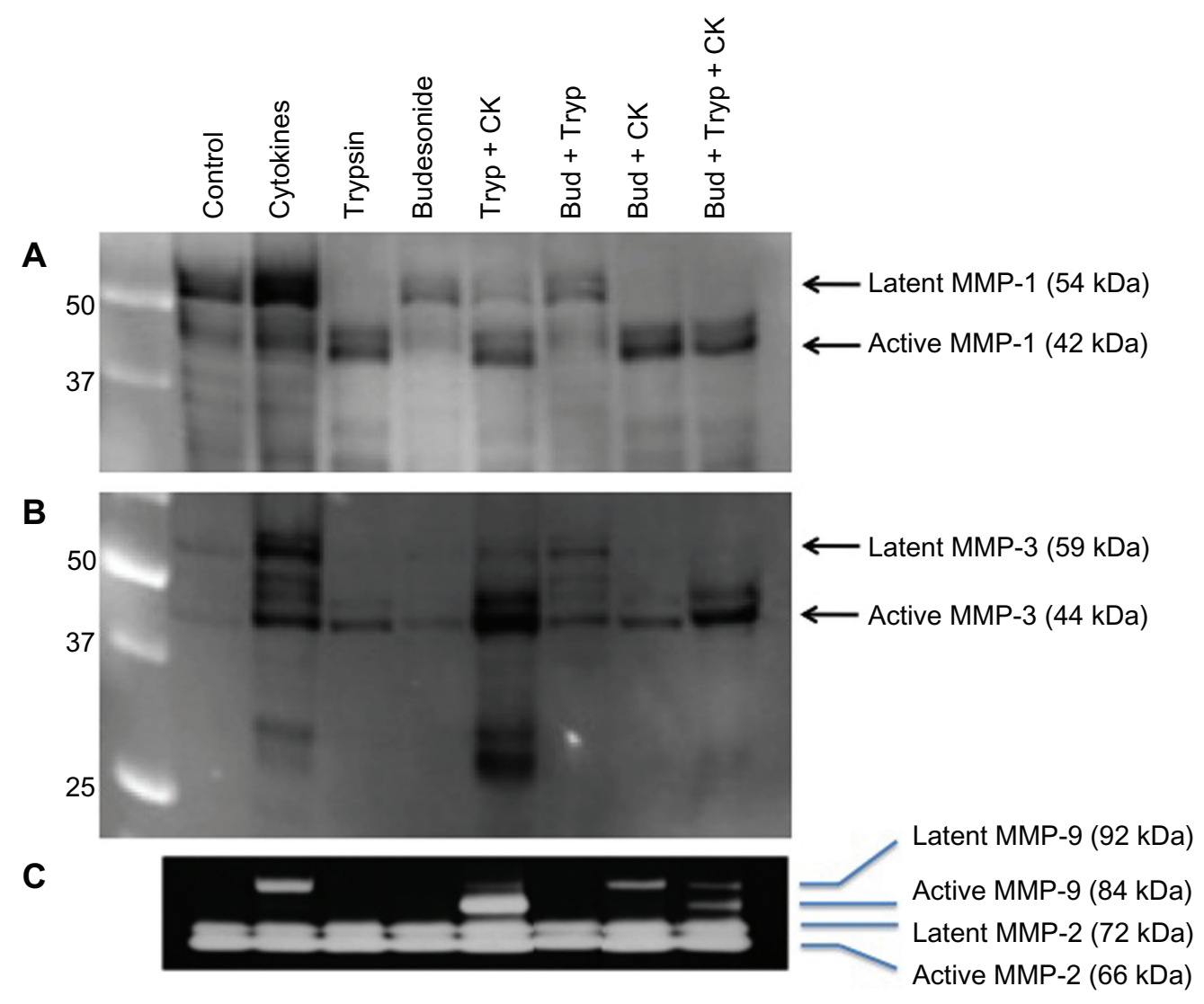

Figure 4 Budesonide inhibits activation of MMPs by trypsin. Fibroblasts were cast into collagen gels and maintained in serum free Dulbecco's Modified Eagle's Medium with or without cytokines, trypsin, and budesonide. After 3 days, the media were harvested and used to assess MMP release. Immunoblottings for MMP-I (A) and MMP-3 (B). (C) Gelatin zymography for MMP-2 and MMP-9.

Notes: The clear bands indicate gelatinase activity. The labels indicate the expected positions for the latent and active forms of the MMPs. The left-most lane in Panels A and B shows the molecular weight standards. The data presented are one representative of three separate experiments.

Abbreviations: Bud, budesonide $100 \mathrm{nM}$; CK, cytokines (interleukin- $1 \beta$ + tumor necrosis factor- $\alpha ; 5 \mathrm{ng} / \mathrm{mL}$ for each); Tryp, trypsin $0.25 \mu \mathrm{g} / \mathrm{mL}$; MMP, metalloproteinase.

In this regard, trypsin or budesonide alone slightly augmented contraction (note the lines for trypsin and budesonide are nearly completely superimposed in Figure 5A). Trypsin plus budesonide minimally enhanced gel contraction compared with each added alone (Figure 5A). Cytokines, as expected, inhibited contraction. When trypsin was added together with cytokines, it had little effect on gel size for the first 24 hours. However, after 24 hours, the combination of trypsin and cytokines resulted in a dramatic decrease in gel size. Importantly, the gel did not reach a stable size. Rather, the gels diminished until they were minimally present after 3 days and were barely detectable by day 4 , consistent with degradation of the collagen gels (Figure 5A). Budesonide significantly delayed the destruction of the collagen gels that occurred in the presence of trypsin and cytokines (Figure 5A). This effect was easily observed on day 2 and was most remarkable on day 3. By day 4 , the gels incubated in the presence of budesonide had also disappeared (Figure 5A).
In order to confirm that rapid decrease of gel size resulted from collagen gel degradation, hydoxyproline, a measure of collagen content, was determined in the gels after 3 days of culture. Cytokines, trypsin, and budesonide alone had no effect on the hydroxyproline content of the gels (Figure 5B). Similarly, there was no effect on hydroxyproline content when budesonide was added together with either cytokines or trypsin. In contrast, when the cytokines were added together with trypsin, the hydroxyproline content was decreased by about $50 \%$ under the assay conditions and time assessed, indicating collagen degradation (Figure 5B). Budesonide significantly and almost completely blocked the ability of cytokines plus trypsin to induce collagen degradation as evidenced by the higher amount of hydroxyproline recovered from the gels (Figure 5B).

Next, in order to determine the specificity of the budesonide effect on collagen degradation, key experiments were repeated with dexamethasone $1 \mu \mathrm{M}$. Consistent with the effect of budesonide, dexamethasone reduced cytokine-induced 
A Gel size

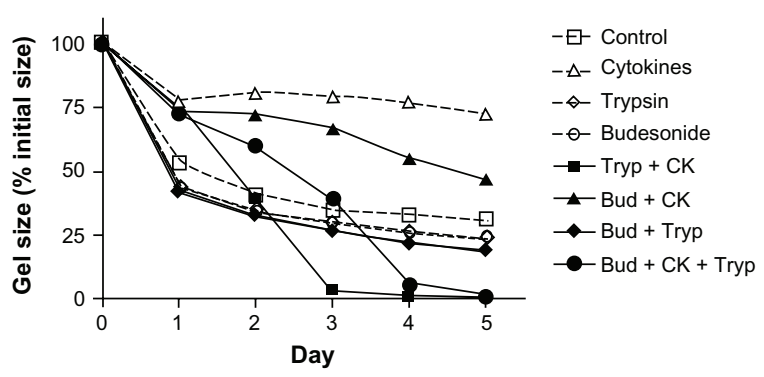

B Hydroxyproline

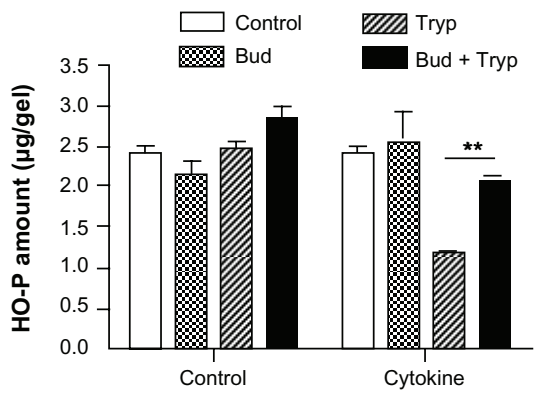

Figure $\mathbf{5}$ Effect of budesonide on collagen gel degradation induced by cytokines plus trypsin. Fibroblasts were cast into three-dimensional collagen gels and maintained in floating culture for 5 days in the presence or absence of cytokines, trypsin, and budesonide. (A) Collagen gel contraction assay. Vertical axis shows gel size expressed as percent of initial size (\%); horizontal axis shows time (day). (B) Hydroxyproline assay. In replicated experiments, gels were harvested on day 3 and hydroxyproline amount was determined. Vertical axis shows hydroxyproline amount expressed as $\mu \mathrm{g} / \mathrm{gel}$; horizontal axis shows media with or without cytokines.

Notes: The data presented in both panels are the mean \pm standard deviation of three separate experiments, each of which included triplicate gels for each condition. ** $P<0.0$ I by one-way analysis of variance followed by Tukey's test for the comparison of trypsin with trypsin + budesonide in the presence of cytokines.

Abbreviations: CK, cytokines (interleukin- $\mathrm{\beta}+$ tumor necrosis factor- $\alpha ; 5 \mathrm{ng} / \mathrm{mL}$ each); Bud, budesonide $100 \mathrm{nM}$; Tryp, trypsin $0.25 \mu \mathrm{g} / \mathrm{mL}$; HO-P, hydroxyproline.

release of MMP-1, MMP-3, and MMP-9, and reduced the trypsin-mediated activation of cytokine-induced MMPs (Figure 6A-C, respectively). Further, dexamethasone, consistent with budesonide, also inhibited cytokine-induced expression of MMP mRNAs (Figure 7). Like budesonide, dexamethasone alone had no effect on collagen gel size, but significantly blocked collagen degradation in the presence of cytokines plus trypsin (data not shown).

\section{Discussion}

The current study demonstrates that budesonide can modify human lung fibroblast-mediated collagen gel contraction and degradation. Budesonide alone slightly augmented fibroblastmediated collagen gel contraction under control conditions. Inflammatory cytokines (IL-1 $\beta$ and TNF $\alpha$ ) significantly inhibited collagen gel contraction and induced MMP production that, after activation, could lead to degradation of the collagen gels. The effects of these cytokines were significantly blocked by budesonide in a concentration-dependent manner. The mechanism for inhibition of collagen degradation is reduced gene expression, release, and subsequent activation of MMPs. These findings suggest that budesonide modifies airway tissue remodeling by regulating MMPs at various levels, ie, synthesis, release, and activation.

Following airway inflammation, airway tissue repair and remodeling occurs. In vitro, fibroblast-mediated contraction and degradation of extracellular matrix have been used as models of tissue remodeling. Many factors can regulate fibroblast-mediated collagen gel contraction and degradation. In this regard, we have previously reported that inflammatory cytokines, IL-1 $\beta$ and TNF $\alpha$, not only modulate collagen gel contraction through stimulating prostaglandin $\mathrm{E}_{2}$ release, ${ }^{24}$ but also stimulate MMP release by fibroblasts. ${ }^{16,25}$ In the current study, IL-1 $\beta$ and TNF $\alpha$ were used to stimulate MMP synthesis and release by the fibroblasts and the effect of budesonide on collagen gel contraction and degradation was investigated. Consistent with our previous reports, budesonide alone slightly augmented collagen gel contraction under control conditions, an effect that is due to inhibition of prostaglandin $\mathrm{E}_{2}$ release. ${ }^{21}$ More importantly, when trypsin was added to activate the MMPs, leading to degradation of the collagenous matrix, the presence of budesonide resulted in less active MMP-9 and MMP-3 and slower degradation of the extracellular collagenous gels.

Matrix metalloproteinases play an important role in the degradation of extracellular matrix. This family of enzymes, which includes 24 members in man, is characterized by having zinc at the active site. ${ }^{26}$ The activity of MMPs is regulated at a number of levels. First, synthesis of MMPs is regulated at the level of mRNA expression as well as by post-translational mechanisms. Second, most of the MMPs are released as latent forms and are activated by proteolytic cleavage that can occur in a stepwise manner. Third, activated MMPs can be inhibited by TIMPs, which is a family of endogenous inhibitors. In the current study, corticosteroids, budesonide and dexamethasone, inhibited not only mRNA expression of MMP-1, MMP-3, and MMP-9, but also inhibited protein release and, to some extent, activation of MMPs. In this context, serine protease activation of MMPs is mediated both by direct proteolytic activation of MMPs and by cell-dependent mechanisms. ${ }^{19}$ While not evaluated in the current study, the latter is more likely to be directly affected by corticosteroids, in that TIMP-1 production by fibroblasts was not significantly altered by corticosteroids. 


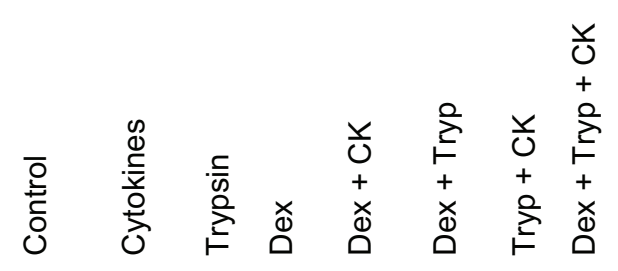

A

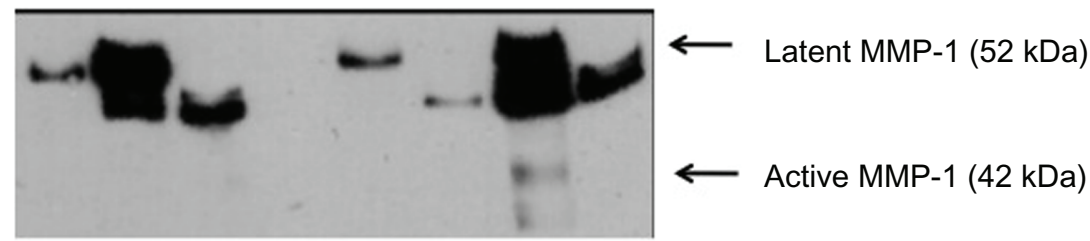

B

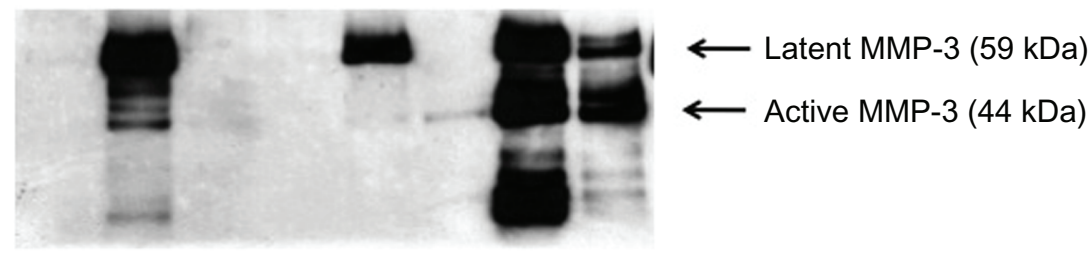

C

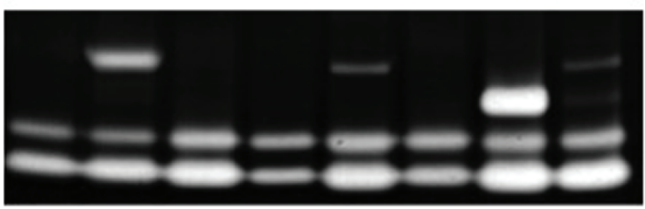

$\longleftarrow$ Latent MMP-9 (92 kDa)

$\longleftarrow$ Active MMP-9 (84 kDa)

$\longleftarrow$ Latent MMP-2 (72 kDa)

$\longleftarrow$ Active MMP-2 (66 kDa)

Figure 6 Effect of dexamethasone on MMP production and activation. Gels were cultured in various conditions (as indicated in the lanes) for 5 days. Media were harvested for gelatin zymography and immunoblotting. (A) Immunoblottings for MMP-I and (B) MMP-3. (C) Gelatin zymography for MMP-2 and MMP-9.

Notes: Arrows indicate expected positions of latent and active forms of the MMPs. Data presented are one representative of three separate experiments.

Abbreviations: CK, cytokines (interleukin- $1 \beta$ + tumor necrosis factor- $\alpha ; 5 \mathrm{ng} / \mathrm{mL}$ each); Dex, dexamethasone I $\mu \mathrm{M}$; Tryp, trypsin $0.25 \mu \mathrm{g} / \mathrm{mL}$; MMP, metalloproteinase.
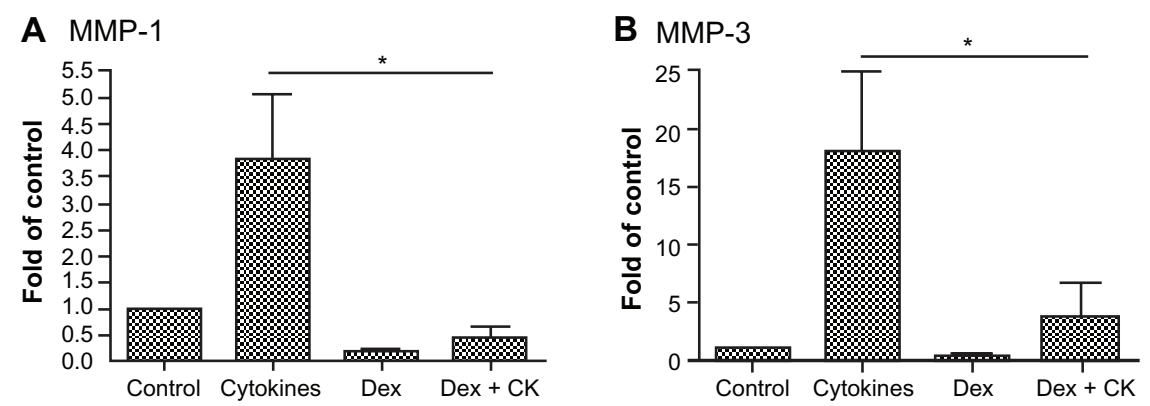

\section{MMP-9}

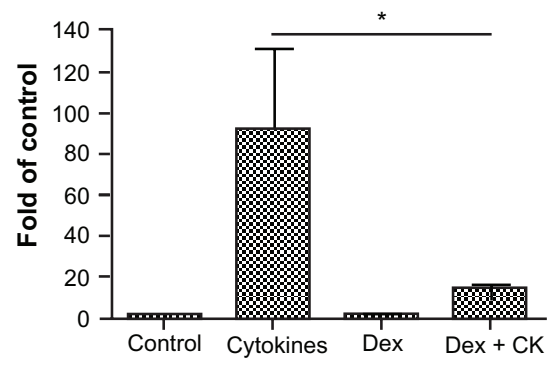

Figure 7 Inhibition of MMP mRNA expression by dexamethasone. Cells were cast into three-dimensional collagen gels and maintained in serum-free Dulbecco's Modified Eagle's Medium with or without cytokines and/or dexamethasone for 3 days. Total RNA was extracted and real-time reverse transcriptase polymerase chain reaction was performed as described in the Methods section for MMP-I (A), MMP-3 (B), and MMP-9 (C).

Notes: The vertical axis shows mRNA level expressed as fold of control (serum-free Dulbecco's Modified Eagle's Medium condition); the horizontal axis shows treatment. Data are presented as the mean \pm standard deviation for three separate experiments. $* P<0.05$ by one-way analysis of variance followed by Tukey's test for the comparison of cytokines with dexamethasone + cytokines.

Abbreviations: CK, cytokines (IL-I $\beta+\mathrm{TNF} \alpha ; 5 \mathrm{ng} / \mathrm{mL}$ each); Dex, dexamethasone I $\mu \mathrm{M} ;$ MMP, metalloproteinase. 


\section{Conclusion}

In summary, in the current study, we demonstrate that budesonide and dexamethasone inhibit degradation of collagenous matrix induced by cytokines in the presence of a protease activator. The mechanism of this effect is inhibition of release of MMPs by cytokine-stimulated human lung fibroblasts as well as decreased activation of MMPs. These findings suggest that inhaled budesonide may modify airway tissue remodeling by regulating MMP release and activation. This may be particularly important in inflammatory diseases characterized by tissue destruction and/or remodeling, such as COPD.

\section{Acknowledgment}

We appreciate the secretarial support of Lillian Richards in this research.

\section{Disclosure}

The authors report no conflicts of interest in this work.

\section{References}

1. Rennard SI, Togo S, Holz O. Cigarette smoke inhibits alveolar repair: a mechanism for the development of emphysema. Proc Am Thorac Soc. 2006;3:703-708.

2. Nagase H, Visse R, Murphy G. Structure and function of matrix metalloproteinases and TIMPs. Cardiovasc Res. 2006;69:562-573.

3. Zheng $T$, Zhu Z, Wang Z, et al. Inducible targeting of IL-13 to the adult lung causes matrix metalloproteinase- and cathepsin-dependent emphysema. J Clin Invest. 2000;106:1081-1093.

4. Han Z, Junxu, Zhong N. Expression of matrix metalloproteinases MMP-9 within the airways in asthma. Respir Med. 2003;97:563-567.

5. Suzuki R, Miyazaki Y, Takagi K, Torii K, Taniguchi H. Matrix metalloproteinases in the pathogenesis of asthma and COPD: implications for therapy. Treat Respir Med. 2004;3:17-27.

6. Li MJ, Huang CX, Okello E, Yanhong T, Mohamed S. Treatment with spironolactone for 24 weeks decreases the level of matrix metalloproteinases and improves cardiac function in patients with chronic heart failure of ischemic etiology. Can J Cardiol. 2009;25:523-526.

7. Haruyama T, Ajioka I, Akaike T, Watanabe Y. Regulation and significance of hepatocyte-derived matrix metalloproteinases in liver remodeling. Biochem Biophys Res Commun. 2000;272:681-686.

8. Olson MW, Bernardo MM, Pietila M, et al. Characterization of the monomeric and dimeric forms of latent and active matrix metalloproteinase- 9 . Differential rates for activation by stromelysin 1. J Biol Chem. 2000;275: 2661-2668.

9. Gueders MM, Foidart JM, Noel A, Cataldo DD. Matrix metalloproteinases (MMPs) and tissue inhibitors of MMPs in the respiratory tract: potential implications in asthma and other lung diseases. Eur J Pharmacol. 2006;533:133-144.
10. Grinnell F. Fibroblast biology in three-dimensional collagen matrices. Trends Cell Biol. 2003;13:264-269.

11. Bell E, Ivarsson B, Merrill C. Production of a tissue-like structure by contraction of collagen lattices by human fibroblasts of different proliferative potential in vitro. Proc Natl Acad Sci U S A. 1979;76: 1274-1278.

12. Wen FQ, Skold CM, Liu XD, et al. Glucocorticoids and TGFbeta1 synergize in augmenting fibroblast mediated contraction of collagen gels. Inflammation. 2001;25:109-117.

13. Mio T, Liu XD, Adachi Y, et al. Human bronchial epithelial cells modulate collagen gel contraction by fibroblasts. Am J Physiol. 1998;274:L119-L126.

14. Liu X, Wen FQ, Kobayashi T, et al. Smad3 mediates the TGF-betainduced contraction of type I collagen gels by mouse embryo fibroblasts Cell Motil Cytoskeleton. 2003;54:248-253.

15. Mio T, Adachi Y, Romberger DJ, et al. Human bronchial epithelial cells modulate collagen gel contraction by fibroblasts. Am J Respir Crit Care Med. 1995;151:A561.

16. Zhu YK, Liu X, Ertl RF, et al. Retinoic acid attenuates cytokine-driven fibroblast degradation of extracellular matrix in three-dimensional culture. Am J Respir Cell Mol Biol. 2001;25:620-627.

17. Togo $\mathrm{S}, \mathrm{Holz} \mathrm{O}$, Liu $\mathrm{X}$, et al. Lung fibroblast repair functions in patients with chronic obstructive pulmonary disease are altered by multiple mechanisms. Am J Respir Crit Care Med. 2008;178:248-260.

18. Zhu Y, Skold CM, Liu X, et al. Fibroblasts and monocyte macrophages contract and degrade three-dimensional collagen gels in extended co-culture. Respir Res. 2001;2:295-299.

19. Fang Q, Liu X, Al-Mugotir M, et al. Thrombin and TNF-alpha/IL-1 beta synergistically induce fibroblast-mediated collagen gel degradation. $\mathrm{Am}$ J Respir Cell Mol Biol. 2006;35:714-721.

20. Kamio K, Sato T, Liu X, et al. Prostacyclin analogs stimulate VEGF production from human lung fibroblasts in culture. Am J Physiol Lung Cell Mol Physiol. 2008;294:L1226-L1232.

21. Skold CM, Liu XD, Zhu YK, et al. Glucocorticoids augment fibroblastmediated contraction of collagen gels by inhibition of endogenous PGE production. Proc Assoc Am Physicians. 1999;111:249-258.

22. Dumin JA, Dickeson SK, Stricker TP, et al. Pro-collagenase-1 (matrix metalloproteinase-1) binds the alpha(2)beta(1) integrin upon release from keratinocytes migrating on type I collagen. $J$ Biol Chem. 2001;276:29368-29374.

23. Loffek S, Schilling O, Franzke CW. Series "matrix metalloproteinases in lung health and disease": biological role of matrix metalloproteinases: a critical balance. Eur Respir J. 2011;38:191-208.

24. Zhu YK, Liu XD, Skold MC, et al. Cytokine inhibition of fibroblastinduced gel contraction is mediated by $\operatorname{PGE}(2)$ and NO acting through separate parallel pathways. Am J Respir Cell Mol Biol. 2001;25:245-253.

25. Fang $Q$, Liu $X, A b e S$, et al. Thrombin induces collagen gel contraction partially through PAR1 activation and PKC-epsilon. Eur Respir J. 2004;24 918-924.

26. Massova I, Kotra LP, Fridman R, Mobashery S. Matrix metalloproteinases: structures, evolution, and diversification. FASEB J. 1998;12: 1075-1095.
Journal of Inflammation Research

\section{Publish your work in this journal}

The Journal of Inflammation Research is an international, peer-reviewed open-access journal that welcomes laboratory and clinical findings on the molecular basis, cell biology and pharmacology of inflammation including original research, reviews, symposium reports, hypothesis formation and commentaries on: acute/chronic inflammation; mediators of inflamma-

\section{Dovepress}

tion; cellular processes; molecular mechanisms; pharmacology and novel anti-inflammatory drugs; clinical conditions involving inflammation. The manuscript management system is completely online and includes a very quick and fair peer-review system. Visit http://www.dovepress.com/ testimonials.php to read real quotes from published authors. 